\title{
How to Write a Scientific Paper
}

\author{
E. Robert Schulman \\ Charlottesville, Virginia
}

\begin{abstract}
We (meaning I) present observations on the scientific publishing process which (meaning that) are important and timely in that unless I have more published papers soon, I will never get another job. These observations are consistent with the theory that it is difficult to do good science, write good scientific papers, and have enough publications to get future jobs.
\end{abstract}

\section{Introduction}

Scientific papers (e.g., Schulman 1988; Schulman \& Fomalont 1992; Schulman, Bregman, \& Roberts 1994; Schulman \& Bregman 1995; Schulman 1996) are an important--though poorly understood--method of publication. They are important because without them scientists cannot get money from the government or from universities. They are poorly understood because they are not written very well (see, for example, Schulman 1995 and selected references therein). An excellent example of the latter phenomenon occurs in most introductions, which are supposed to introduce the reader to the subject so that the paper will be comprehensible even if the reader has not done any work in the field.

The real purpose of introductions, of course, is to cite your own work (e.g., Schulman et al. 1993a), the work of your advisor (e.g., Bregman, Schulman, \& Tomisaka 1995), the work of your spouse (e.g., Cox, Schulman, \& Bregman 1993), the work of a friend from college (e.g., Taylor, Morris, \& Schulman 1993), or even the work of someone you've never met, as long as your name happens to be on the paper (e.g., Richmond et al. 1994). Note that these citations should not be limited to refereed journal articles (e.g., Collura et al. 1994), but should also include conference proceedings (e.g., Schulman et al. 1993b), and other published or unpublished work (e.g., Schulman 1990).

At the end of the introduction you must summarize the paper by reciting the section headings. In this paper, we discuss scientific research (section 2), scientific writing (section 3), scientific publication (section 4), and draw some conclusions (section 5).

\section{Scientific Research}

The purpose of science is to get paid for doing fun stuff (Schulman et al. 1991). Nominally, science involves discovering something new about the Universe, but this isn't really necessary. What is really necessary is a grant. In order to obtain a grant, your application must state that the research will discover something incredibly fundamental. The grant agency must also believe that you are the best person to do this particular research, so you should cite yourself both early (Schulman 1994) and often (Schulman et al. 1993c). Feel free to cite other papers as well (e.g., Blakeslee et al. 1993; Levine et al. 1993), so long as you are on the author list.

Once you get the grant, your university, company, or government agency will immediately take 30 to $70 \%$ of it so that they can heat the building, pay for Internet, and purchase large yachts. 
Now it's time for the actual research. You will quickly find out that (a) your project is not as simple as you thought it would be and (b) you can't actually solve the problem. However -- and this is very important -- you must publish anyway (Schulman \& Bregman 1994).

\section{Scientific Writing}

You've spent years on a project and have finally discovered that you can't solve the problem you set out to solve. Nonetheless, you have a responsibility to present your research to the scientific community (Schulman et al. 1993d). Be aware that negative results can be just as important as positive results, and also that if you don't publish enough you'll never be able to stay in science.

While writing a scientific paper, the most important thing to remember is that the word "which" should almost never be used. Be sure to spend at least $50 \%$ of your time (i.e., 12 hours a day) typesetting the paper so that all the tables look nice (Schulman \& Bregman 1992).

\section{Scientific Publishing}

You've written the paper, and now it's time to submit it to a scientific journal. The journal editor will pick the referee most likely to be offended by your paper, because then at least the referee will read it and get a report back within the lifetime of the editor. Referees who don't care one way or the other about a paper have a tendency to leave manuscripts under a growing pile of paper until the floor collapses, killing the 27 English graduate students who share the office below (Schulman, Cox, \& Williams 1993).

Be aware that every scientific paper contains serious errors. If your errors are not caught before publication, you'll eventually have to write an erratum to the paper explaining (a) how and why you messed up and (b) that even though your experimental results are now totally different, your conclusions need not be changed. Errata can be good for your career. They are easy to write, and the convention is to reference them as if they were real papers, leading the casual reader--and perhaps also the Science Citation Index--to think that you've published more papers than you really have ( $\underline{\text { Schulman et al. 1994) }}$.

\section{Conclusions}

The conclusion section is very easy to write: all you have to do is to take your abstract and change the tense from present to past. It's considered good form to mention at least one relevant theory only in the abstract and conclusion. By doing this, you don't have to say why your experiment does (or does not) agree with the theory, you merely have to state that it does (or does not).

We (meaning I) presented observations on the scientific publishing process which (meaning that) are important and timely in that unless I have more published papers soon, I will never get another job. These observations are consistent with the theory that it is difficult to do good science, write good scientific papers, and have enough publications to get future jobs.

\section{References}

- Blakeslee, J., Tonry, J., Williams, G.V., \& Schulman, E. 1993 Aug 2, Minor Planet Circular 22357

- Bregman, J.N., Schulman, E., \& Tomisaka, K. 1995, Astrophysical Journal, 439, 155

- Collura, A., Reale, F., Schulman, E., \& Bregman, J.N. 1994, Astrophysical Journal, 420, L63

- Cox, C. V., Schulman, E., \& Bregman, J.N. 1993, NASA Conference Publication 3190, 106

- Levine, D.A., Morris, M., Taylor, G.B., \& Schulman, E. 1993, Bulletin of the American Astronomical Society, 25, 1467 
- Richmond, M.W., Treffers, R.R., Filippenko, A.V., Paik, Y., Leibundgut, B, Schulman, E., \& Cox, C.V. 1994, Astronomical Journal, 107, 1022

- Schulman, E. 1988, Journal of the American Association of Variable Star Observers, 17, 130

- Schulman, E. 1990, Senior thesis, UCLA

- Schulman, E. 1994, Bulletin of the American Astronomical Society, 26, 1411

- Schulman, E. 1995, Ph.D. thesis, University of Michigan

- Schulman, E. 1996, Publications of the Astronomical Society of the Pacific, 108, 460

- Schulman, E., Bregman, J.N., Collura, A., Reale, F., \& Peres, G. 1993a, Astrophysical Journal, 418, L67

- Schulman, E., Bregman, J.N., Collura, A., Reale, F., \& Peres, G. 1994, Astrophysical Journal, $\underline{\text { 426, L55 }}$

- Schulman, E. \& Bregman, J.N. 1992, Bulletin of the American Astronomical Society, 24, 1202

- Schulman, E. \& Bregman, J.N. 1994, in The Soft X-Ray Cosmos, ed. E. Schlegel \& R. Petre (New York: American Institute of Physics), 345

- Schulman, E. \& Bregman, J.N. 1995, Astrophysical Journal, 441, 568

- Schulman, E., Bregman, J.N., Brinks, E., \& Roberts, M.S. 1993b, Bulletin of the American. Astronomical Society, 25, 1324

- Schulman, E., Bregman, J.N., \& Roberts, M.S. 1994, Astrophysical Journal, 423, 180

- Schulman, E., Bregman, J.N., Roberts, M.S., \& Brinks, E. 1991, Bulletin of the American Astronomical Society, 23, 1401

- Schulman, E., Bregman, J.N., Roberts, M.S., \& Brinks, E. 1993c, NASA Conference Publication. $\underline{3190,199}$

- $\underline{\text { Schulman, E., Bregman, J.N., Roberts, M.S., \& Brinks, E. 1993d, Astronomical Gesellschaft }}$ Abstract Series 8, 141

- Schulman, E., Cox, C.V., \& Williams, G.V. 1993 June 4, Minor Planet Circular 22185

- Schulman, E. \& Fomalont, E.B. 1992, Astronomical Journal, 103, 1138

- Taylor, G.B., Morris, M., \& Schulman, E. 1993, Astronomical Journal, 106, 1978 